'Laboratôrio de Genômica, Instituto Agronômico de Pernambuco (IPA), Avenida General San Martin, 1371 Bongi, CEP 50761-000, Recife, PE, Brasil

2Laboratório de Controle Biolōgico, Instituto Agronômico de Pernambuco (IPA), Recife, PE, Brasil ${ }^{3}$ Bolsista de Pós-Doutorado PNPD/ CAPES/FINEP-IPA

*autor correspondente 凶adalia.mergulhao@ipa.br

\title{
Caracterização filogenética de isolados de Beauveria bassiana originados de diferentes insetos hospedeiros
}

\author{
Phylogenetic characterization of Beauveria bassiana isolates \\ originating from different host insects
}

Adălia Cavalcanti do Espírito Santo Mergulhão ${ }^{*}$, Maria Luiza Ribeiro Bastos da Silva ${ }^{1,3}$, Vanildo Alberto Leal Bezerra Cavalcanti², Maria do Carmo Catanho Pereira de Lyra'

RESUMO: Este trabalho objetivou avaliar a variabilidade genética usando a região do espaço interno transcrito (ITS) do DNA ribossomal (rDNA) e a filogenia de cinco isolados de Beauveria bassiana: IPA145, IPA148, IPA223, IPA225 e IPA226, obtidos de diferentes hospedeiros e origens geográficas, provenientes do Laboratório de Controle Biológico do Instituto Agronômico de Pernambuco - IPA. A região ITS foi amplificada usando os primers ITS1 e ITS4, em que foi observado um amplicon com tamanho médio de $800 \mathrm{pb}$, o qual foi sequenciado. As sequências de DNA dos isolados de Beauveria foram comparadas com as sequências do banco de dados (GenBank). Os resultados mostram que todos os isolados, com exceção do IPA145, apresentaram-se como grupo monofilético. O IPA145 apresentou uma identidade genética com Cordyceps bassiana e B. bassiana de $99 \%$. O IPA148 foi o mais distante geneticamente com relação aos demais, com menos de $40 \%$ de similaridade. A maioria dos isolados de Beauveria estudados mostrou-se distinta filogeneticamente.

PALAVRAS-CHAVE: Fungo entomopatogênico, filogenia, ITS, Cordyceps, rDNA.
ABSTRACT: This study aimed to evaluate genetic variability using the internal transcribed spacer (ITS) region of ribosomal DNA ( $r D N A)$ and the phylogeny of five Beauveria bassiana: IPA145, IPA148, IPA223, IPA225 and IPA226, obtained from different hosts and geographical origin from the Biological Control Laboratory of the Agronomic Institute of Pernambuco - IPA. The ITS region was amplified using primers ITS1 and ITS4, where an amplicon with average size of $800 \mathrm{bp}$ was observed and sequenced. The DNA sequences of Beauveria isolates were compared with sequences in the database (GenBank). The results show that all isolates, except IPA145, were presented as a monophyletic group. IPA145 showed a genetic identity of $99 \%$ with Cordyceps bassiana and B. bassiana. IPA148 was the most genetically distant from the others, with less than $40 \%$ similarity. Most of the Beauveria isolates studied proved to be phylogenetically distinct.

KEYWORDS: Entomopathogenic fungus, phylogeny, ITS, Cordyceps, rDNA.

Beauveria bassiana Bals. Vuill. (Deuteromycota: Hyphomycetes) é um fungo entomopatogênico, que existe naturalmente nos solos. É de grande interesse no controle biológico de diversas pragas agrícolas, o que lhe confere importância econômica no Brasil. Destaca-se por apresentar um amplo espectro de insetos hospedeiros, que podem ser controlados por este fungo (XIAO et al., 2012; 
ALVES et al., 2012). Por possuir uma grande vantagem sobre os pesticidas convencionais, devido à sua persistência na população hospedeira, por reduzir a longevidade e ocasionar altas taxas de mortalidade em larvas e adultos das populações de insetos, torna-se um grande aliado para o uso como controle biológico (ALVES, 1998; ROBERTS; CASTILLO, 1980). Outra grande vantagem do uso de fungos entomopatogênicos é a sua resistência a alguns pesticidas, como estudado por Kaaya e Hassan (2000), que observaram que estes fungos reduzem a frequência do uso de acaricida químico, bem como diminuem a necessidade de tratamentos de doenças causadas pelo carrapato. A B. bassiana, além de ser de fácil dispersão, possui grande variedade de hospedeiros (Coleoptera, Lepidoptera, Hemiptera, Diptera, Hymenoptera e Orthoptera). Segundo Rehner e Buckley (2005), as espécies de B. bassiana que infectam apenas um hospedeiro é chamada de sensu stricto e aquelas que infectam mais de um hospedeiro é chamada de sensu lato. Já Rehner et al. (2011) concluíram, através de análise dos genes RNA polimerase I da subunidade grande (RPB1), RNA polimerase II da subunidade grande (RPB2) e subunidade alfa do factor de alongamento (TEF), que $B$. bassiana é um agregado de cinco espécies, tais como $B$. bassiana sensu stricto, B. varroae, B. kipukae, B. pseudobassiana e B. sungu. Fernandes et al. (2006), trabalhando com vários isolados de Beauveria de diferentes regiões geográficas, verificaram diferenças genéticas quando utilizaram o marcador da região ITS. Beauveria apresenta atividades de proteases e quitinases que hoje são aceitas como fatores determinantes na virulência dos fungos entomopatogênicos, que passam a ter habilidade de hidrolisar os principais polímeros constituintes da cutícula, proteínas e quitina, organizados em camadas denominadas exo e endocutícula (SILVA et al., 2006; SAMISH; GLAZER, 1991; St. LEGER; COOPER; CHARLEY, 1986). Apesar de haver um interesse crescente no uso deste agente de controle biológico, há pouco conhecimento acerca do seu efeito sobre o meio ambiente. As espécies do gênero Beauveria têm sido reportadas por produzir metabólitos secundários, como bassianina, bassiacridina e beauvericina, entre outros (QUESADA-MORAGA; VEY, 2004). Hoje, a grande pergunta com relação ao conceito de espécies morfológicas em Beauveria é se o padrão de variação morfofisiológica tem relação com a filogenia; outra questão é se as espécies morfológicas são cripticamente diversas, ou seja, se estas espécies estão reprodutivamente isoladas umas das outras, apesar de sua morfologia ser muito semelhante (SCHAEFER, 1998).

De acordo com Devi et al. (2001), alguns isolados de Beauveria têm sido avaliados devido ao seu potencial como biopesticidas, porém as características fenotípicas não são suficientes para distinguir os diferentes tipos de isolados estudados (GAITAN et al., 2002). Nesta perspectiva, estudos moleculares envolvendo a técnica da PCR e os clusters gênicos do rDNA (DNA ribossomal) têm facilitado a identificação taxonômica e contribuído para expandir o conhecimento sobre a diversidade e distinguir diferenças entre gêneros e isolados de uma mesma espécie de Beauveria (WADA et al., 2003; COATES; HELLMICH; LEWIS, 2002). Recomenda-se que o fragmento de rDNA a ser amplificado para a análise da diversidade intraespecífica ou entre grupos de isolados com elevada afinidade filogenética deve incluir o espaço interno transcrito (ITS1 e ITS2), uma região que apresenta maior variabilidade tanto na composição de bases quanto no tamanho do fragmento (MENEZES et al., 2010; ROSADO; DUARTE; MENDONÇA-HAGLER, 1999). Segundo Carneiro et al. (2008), a variabilidade genética - inter e intraespecífica - pode ser capaz de identificar e caracterizar isolados de Beauveria. Dessa forma, o trabalho em questão teve como objetivo avaliar a variabilidade genética da região do espaço interno transcrito (ITS) do DNA ribossomal (rDNA) e a filogenia de isolados de $B$. bassiana obtidos de diferentes hospedeiros e origens geográficas distintas, provenientes do Laboratório de Controle Biológico do Instituto Agronômico de Pernambuco - IPA.

Os cinco isolados de Beauveria sensu lato foram obtidos da coleção do Laboratório de Controle Biológico do Instituto Agronômico de Pernambuco - IPA. Cada isolado foi oriundo de hospedeiros diferentes e de regiões geográficas distintas, como descrito a seguir: IPA145 (Coleoptera: Curculionidae; Indeterminado); IPA148 (Coleoptera: Coccinelidae; Indeterminado); IPA223 (Lepidoptera: Pyrilidae, Paraíba-BR); IPA225 (Orthoptera: Acrididae; Recife-PE-BR); IPA226 (Coleoptera: Curculionidae; Cabo de Santo Agostinho-PE-BR). A extração de DNA foi realizada colocando os isolados para crescer em $25 \mathrm{~mL}$ de meio BDA líquido por $72 \mathrm{~h}$, à temperatura ambiente $\left(28^{\circ} \mathrm{C}\right)$, sem agitação. Após este período, $20 \mathrm{~g}$ do micélio foram macerados em nitrogênio líquido, sendo então utilizado o kit de extração de DNA genômico da QIAGEN (DNeasy Blood \& Tissue Kit), conforme metodologia do fabricante. O DNA extraído foi quantificado usando o marcador de massa molecular (Invitrogen); a qualidade e a quantidade do DNA foram visualizadas em gel de agarose a 0,8\% em tampão TBE 0,5X a $100 \mathrm{~V}$ e corado com SybrGold (Invitrogen). A reação de amplificação foi realizada utilizando os primers ITS1 (5‘-TCC GTA GGT GAA CCT GCG G-3‘) e ITS4 (5'-TCC TCC GCT TAT TGA TAT GC-3`) (WHITE et al., 1990). Os fragmentos de DNA amplificados foram separados em gel de agarose a 1,5\%, tampão TBE $0,5 \mathrm{X}$ e corados com SybrGold em corrente elétrica de $100 \mathrm{~V}$, sendo visualizados sob luz ultravioleta e fotografados em fotodocumentador (LPIX-Loccus Brasil). Os fragmentos de ITS1 e ITS4 amplificados foram purificados usando a metodologia de acetato de amônio a 7M (LYRA, 2001). O sequenciamento do DNA foi realizado na plataforma do Centro Nacional de Recursos Genéticos (CENARGEN-EMBRAPA). As sequências direta e reversa foram alinhadas no programa BioEdit v.7.0.0, e foi realizada a análise de reconstrução filogenética com 0 programa MEGA v.4.0 (TAMURA; NEI; KUMAR, 2004), usando o método Neighbor-Joining (SAITOU; NEI, 1987) com teste de filogenia Bootstrap 1000 repetições, gaps com deleção Pairwise e modelo de nucleotídeo: Maximum Composite Likelihood Tamura-Nei. Sequências do banco de dados de genes (GenBank, http://www.ncbi.nlm.nih.gov) foram utilizadas para auxiliar as análises fenéticas com base nas sequências de nucleotídeos obtidas. 


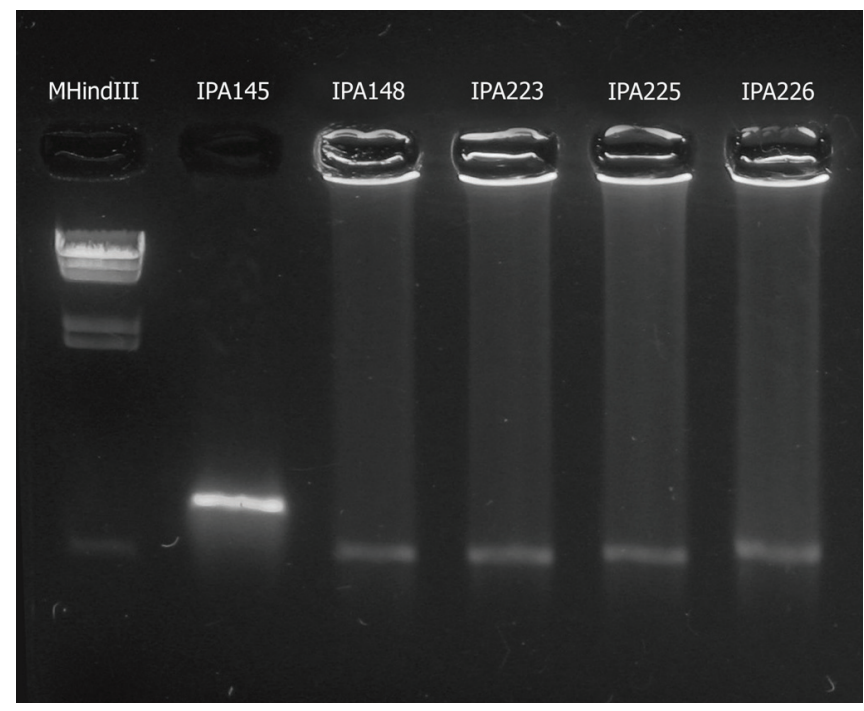

Figura 1. Amplificação da região ITS do rDNA de isolados de Beauveria (IPA145, IPA148, IPA223, IPA225 e IPA226) obtidos da coleção do Laboratório de Controle Biológico do IPA, Marcador (M): HindIII pb Ladder.
O tamanho dos fragmentos amplificados dos cincos isolados de fungos a partir da utilização dos iniciadores ITS1 e ITS4 foi de aproximadamente 800 pb, e apenas um amplicon foi gerado. O sequenciamento da região ITS do isolado IPA145 revelou homologia de 99\% com as sequências de B. bassiana depositadas no GenBank (Figuras 1 e 2). Os fragmentos amplificados visualizados na mesma posição no gel de agarose foram considerados de mesmo tamanho. Segundo Carneiro et al. (2004), um fragmento amplificado de $600 \mathrm{pb}$ de isolados de $B$. bassiana foi sequenciado e revelou uma homologia de mais de $97 \%$ com sequências do rDNA de B. bassiana depositadas no GenBank. De acordo com Carneiro et al. (2008), sequências da região ITS do rDNA são capazes de identificar e caracterizar isolados de Beauveria, e são úteis para detectar variabilidade inter e intraespecífica dentro deste gênero. Entretanto, o marcador utilizado neste estudo não diferenciou todos os isolados de Beauveria no nível de espécie. Desta forma, a região ITS foi sequenciada, para uma melhor análise da variabilidade genética entre os grupos

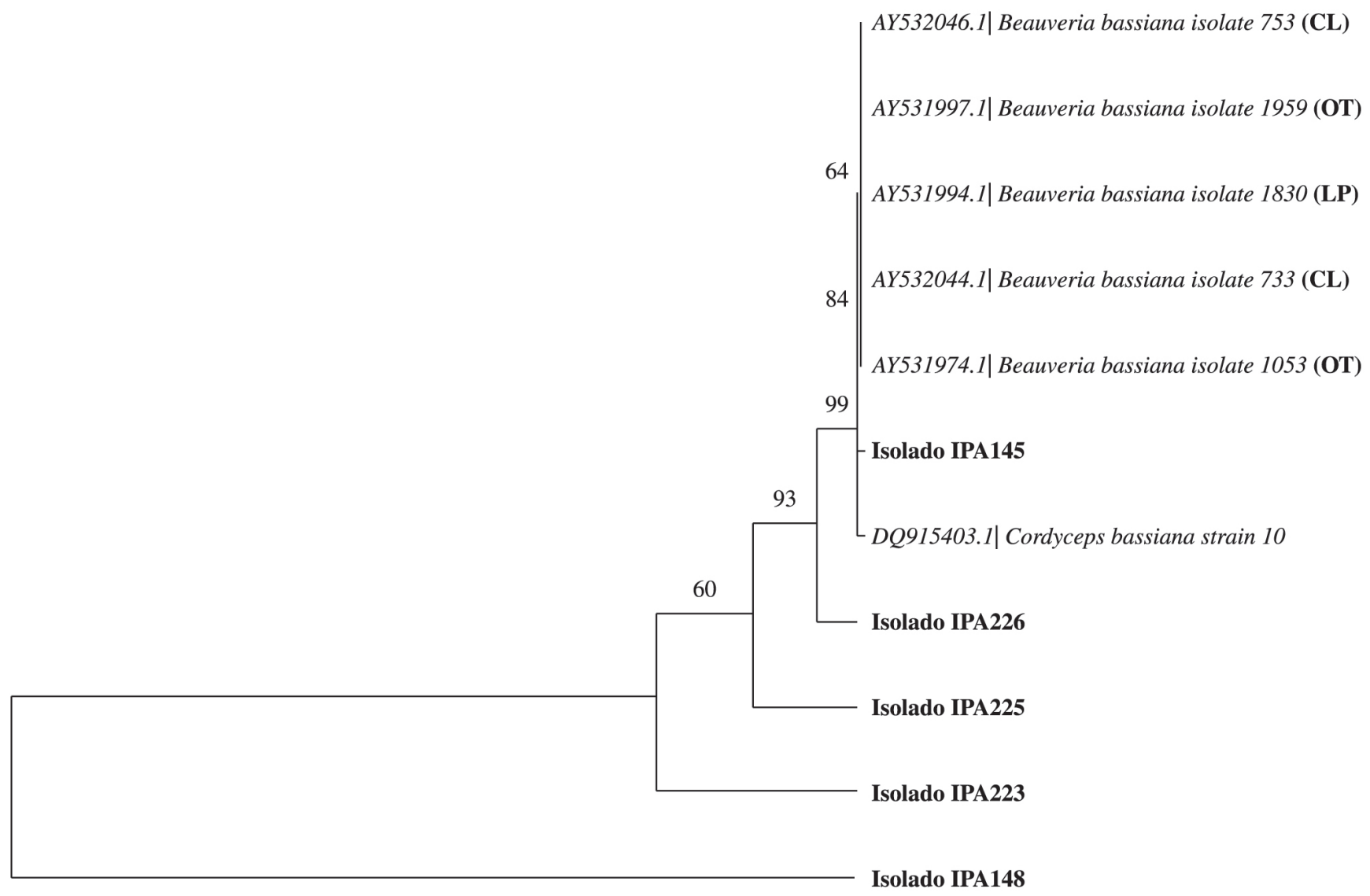

\begin{tabular}{lllllll}
\hline & $\mid$ & & & & & \\
\hline 0.6 & 0.5 & 0.4 & 0.3 & 0.2 & 0.1 & 0.0
\end{tabular}

Figura 2. Árvore filogenética construída com base no sequenciamento da região ITS1 e ITS4 de isolados de Beauveria (IPA145, IPA148, IPA223, IPA225 e IPA226) da coleção do IPA alinhadas pelo programa BioEdit v.7.0 usando Neighbor-Joining (NJ) com Tamurai-Nei e testes de 1000 Bootstrap no programa MEGA v.4.0. 
de isolados de Beauveria estudados. Utilizaram-se sequências do GenBank - AY532046 e AY532044 isoladas de Coleptera (CL); AY531997 e AY531974 isoladas de Orthoptera (OT) e AY531994 isolada de Lepdoptera (LP) - para comparar com as nossas sequências (Figura 2). Os resultados demonstraram que todos os isolados estudados, com exceção do IPA145, apresentaram-se como grupo monofilético, resultado este diferente do encontrado por Rehner e Buckley (2005), que também analisaram a região ITS em isolados de Beauveria coletados no Brasil de diferentes hospedeiros. Usamos, para comparar com os nossos dados, sequências de B. bassiana do GenBank que tinham hospedeiros de mesma Ordem dos insetos. Entretanto, observamos que, neste caso, independentemente de serem isolados de hospedeiros de uma mesma ordem - como Coleoptera, Lepidoptera e Orthoptera -, não houve influência na taxonomia destes isolados, mostrando serem isolados distintos filogeneticamente, exceto para o isolado IPA145. O interessante foi que o IPA145 apresentou uma identidade genética com Cordyceps bassiana de 99\%. O IPA148 foi o mais distante geneticamente com relação aos demais isolados estudados, com menos de $40 \%$ de similaridade. Podemos inferir, diante dos resultados, que a filogenia molecular de Beauveria não está definida, devido ao pouco entendimento taxonômico atual deste gênero. Segundo Rehner e Buckley (2005), que trabalharam com 87 isolados de Beauveria e Cordyceps oriundos de toda parte do mundo, encontrados em diferentes hospedeiros, observou-se que a diversidade filogenética pode indicar uma história de diversificação críptica e que as análises moleculares são ferramentas úteis para a avaliação de espécies, para elucidar a história evolutiva e ecológica destes gêneros.

Podemos dizer que, neste trabalho, as diferentes origens dos fungos estudados e os distintos hospedeiros dos quais foram isolados mostraram uma distância genética entre si e que estudos polifásicos (moleculares, culturais) e genéticos, como as teleomórfas de Cordyceps e anamórfas de Beauveria, podem dar resultados relevantes para futuras pesquisas deste grupo de grande importância, que são os fungos entomopatogênicos. Além do mais, essas informações irão beneficiar futuros estudos moleculares no que diz respeito à interação insetofungo e facilitar o custo-benefício do desenvolvimento de Beauveria como micoinseticidas e biocatalisadores microbianos, resultando em benefícios diretos ao pequeno produtor brasileiro.

\section{Referências}

ALVES, L. F. A. et al. First record of Beauveria bassiana (Hyphomycetes: Moniliales) on adults of cassava lace bug Vatiga manihotae (Drake) (Hemiptera: Tingidae) in Brazil. Arquivos do Instituto Biológico, v. 79, n. 2, p. 309-311, 2012.

ALVES, S. B. Fungos entomopatogênicos. In: ALVES, S. B. (Ed.). Controle microbiano de insetos. Piracicaba: Fealq, 1998. p. 289-381.

CARNEIRO, A. A. et al. Caracterização molecular de fungos entomopatogênicos utilizados no controle biológico de pragas do milho - Beauveria bassiana versus Spodoptera frugiperda. Sete Lagoas: Embrapa Milho e Sorgo, 2004. 10 p. (Embrapa Milho e Sorgo. Comunicado técnico, n. 93).
CARNEIRO, A. A. et al. Molecular characterization and pathogenicity of isolates of Beauveria spp. to fall armyworm. Pesquisa Agropecuária Brasileira, Recife, v. 43, n. 4, p. 513-520, 2008. Disponível em: <http://www.alice.cnptia.embrapa.br/handle/ doc/491183>.

COATES, B. S.; HELLMICH, R. L.; LEWIS, L. C. Beauveria bassiana haplotype determination based on nuclear rDNA internal transcribed spacer PCR-RFLP. Mycological Research, Cambridge, v. 106, p. 40-50, 2002. http://dx.doi.org/10.1017/ S0953756201005305

DEVI, K. U. et al. Laboratory evaluation of the virulence of Beauveria bassiana isolates to the sorghum shoot borer Chilopartellus Swinhoe (Lepidoptera: Pyralidae) and their characterization by RAPD-PCR. World Journal of Microbiology and Biotechnology, Oxford, v. 17, n. 2, p. 131-137, 2001. http:// dx.doi.org/10.1023/A:1016633427739

FERNANDES, E. K. K. et al. Study on morphology, pathogenicity, and genetic variability of Beauveria bassiana isolates obtained from Boophilus microplus tick. Parasitology Research, Berlin, v. 98, p.324-332, 2006. http://dx.doi.org/10.1007/s00436-005-0058-y

GAITAN, A. et al. Genetic variability of Beauveria bassiana associated with the coffee berry borer (Hypothenemus hampei) and other insects. Mycological Research, Cambridge, v. 106, p. 13071314, 2002. http://dx.doi.org/10.1017/S0953756202006676

KAAYA, G.P.; HASSAN, S. Entomogenous fungi as promising biopesticides for tick control. Experimental and Applied Acarology, Amsterdam, v. 24, p. 913-926, 2000. Disponível em: $<$ http://www.nhm.ac.uk/hosted_sites/acarology/saas/e-library/ pdf000200/a000169.pdf>.

LYRA, M. C. C. P. Estudios genéticos y fisiológicos del gen nolT de la región específica de cultivar, nolXWBTUV, de la bacteria de amplio rango de nodulación HH103 y sus implicaciones en el Sistema de Secreción de Tipo III (TTSS). 2001. 159 f. Tese (Doutorado)-Universidad de Sevilla, Espanha, 2001.

MENEZES, J. P. et al. Variabilidade genética na região ITS do rDNA de isolados de Trichoderma spp. (Biocontrolador) e Fusarium oxysporum f. sp. Chrysanthemi. Ciência e Agrotecnologia, Lavras, v. 34, n. 1, p. 132-139, 2010. http://dx.doi.org/10.1590/ S1413-70542010000100017

QUESADA-MORAGA, E.; VEY, A. Bassiacridin, a protein toxic for locusts secreted by the entomopathogenic fungus Beauveria bassiana. Mycological Research, Cambridge, v. 108, p. 441-452, 2004. http://dx.doi.org/10.1017/S0953756204009724

REHNER, S. A.; BUCKLEY, E. A Beauveria phylogeny inferred from nuclear ITS and EF1- $\alpha$ sequences: evidence for cryptic diversification and links to Cordyceps teleomorphs. Mycologia New York, v. 97, n. 1, p. 84-98, 2005. Disponível em: <http://www. mycologia.org/content/97/1/84.full.pdf>.

REHNER, S. A. et al. Phylogeny and systematics of the anamorphic, entomopathogenic genus Beauveria. Mycologia, New York, v. 103, p. 1055-1073, 2011. Disponível em: <http://www.mycologia.org/ content/103/5/1055.full.pdf+html $>$.

ROBERTS, D. W.; CASTILLO, J. M. Bibliography on pathogens of medically important arthropods. Bullitin of the World Health Organization, New York, v. 58, p. 190-197, 1980.

ROSADO, A. S.; DUARTE, G. F.; MENDONÇA-HAGLER, L. C. A moderna microbiologia do solo: Aplicação de técnicas de biologia molecular. In: SIQUEIRA, J. O. et al. (Eds.). Inter-relação fertilidade, biologia do solo e nutrição de plantas. Viçosa: SBCS; Lavras: UFLA/DCS, 1999. p. 429-228. 
SAITOU, N.; NEI, M. The neighbor-joining method: A new method for reconstructing phylogenetic trees. Molecular Biology and Evolution, Chicago, v. 4, n. 4, p. 406-425, 1987. Disponível em: $<$ http://mbe.oxfordjournals.org/content/4/4/406.full.pdf+html $>$.

SAMISH, M.; GLAZER, J. Killing ticks with parasitic nematodes of insects. Journal of Invertebrate Pathology, San Diego, v. 58, p. 281-282, 1991.

SCHAEFER, C. W. Phylogeny, Systematics, and Practical Entomology: The Heteroptera (Hemiptera). Anais da Sociedade Entomológica do Brasil, Jaboticabal, v. 27, n. 4, p. 499-511, 1998. http://dx.doi.org/10.1590/S0301-80591998000400001

SILVA, A. S. et al. Ação do fungo Beauveria bassiana, isolado 986, sobre o ciclo biológico do cascudinho Alphitobius diaperinus em laboratório. Ciência Rural, Santa Maria, v. 36, n. 6, p. 19441947, 2006. http://dx.doi.org/10.1590/S0103-84782006000600047

St. LEGER, R. J., COOPER, R. M.; CHARLEY, A. K. Cuticledegrading enzymes of entomopathogenic fungi: cuticle degradation in vitro by enzymes from entomopathogens. Journal of
Invertebrate Pathology, San Diego, v. 47, n.2, p. 167-177, 1986. http://dx.doi.org/10.1016/0022-2011(86)90043-1

TAMURA, K.; NEI, M.; KUMAR, S. Prospects for inferring very large phylogenies by using the Neighbor-Joining method. Proceedings of the National Academy of Sciences of the USA, Washington, v. 101, n. 30, p. 11030-11035, 2004. Disponível em: <http://www.pna10.1073/pnas.0404206101>.

WADA, S. et al. Discrimination of Japanase isolates of Beauveria brongniartii (Deuteromycotina: Hyphomycetes) by RFLP or the rDNA-ITS regions. Applied Entomology and Zoology, Tokyo, v. 38, p. 551-557, 2003. http://dx.doi.org/10.1303/aez.2003.551

WHITE T. J. et al. Amplification and direct sequencing of fungal ribosomal RNA genes for phylogenetics. In: INNIS, M. A. et al. (Eds.). PCR Protocols: A Guide to Methods and Applications. San Diego: Academic, 1990. p. 315-322. PMid:1696192.

XIAO, G. et al. Genomic perspectives on the evolution of fungal entomopathogenicity in Beauveria bassiana. Scientific Reports, London, v. 483, p. 1-10, 2012. http://dx.doi.org/10.1038/ srep00483(2012). 